\title{
ARTICLE
}

\section{Molecular basis for ligand activation of the human KCNQ2 channel}

\author{
Xiaoxiao $\mathrm{Li}^{1}$, Qiansen Zhang $\mathbb{D}^{2}$, Peipei $\mathrm{Guo}^{2}$, Jie $\mathrm{Fu}^{2}$, Lianghe $\mathrm{Mei}^{3}$, Dashuai $\mathrm{Lv}^{4}$, Jiangqin Wang ${ }^{1}$, Dongwu Lai ${ }^{5}$, Sheng $\mathrm{Ye}^{4,6}$, \\ Huaiyu Yang ${ }^{2}$ and Jiangtao Guo,
}

The voltage-gated potassium channel KCNQ2 is responsible for M-current in neurons and is an important drug target to treat epilepsy, pain and several other diseases related to neuronal hyper-excitability. A list of synthetic compounds have been developed to directly activate KCNQ2, yet our knowledge of their activation mechanism is limited, due to lack of high-resolution structures. Here, we report cryo-electron microscopy (cryo-EM) structures of the human KCNQ2 determined in apo state and in complex with two activators, ztz240 or retigabine, which activate KCNQ2 through different mechanisms. The activator-bound structures, along with electrophysiology analysis, reveal that ztz240 binds at the voltage-sensing domain and directly stabilizes it at the activated state, whereas retigabine binds at the pore domain and activates the channel by an allosteric modulation. By accurately defining ligand-binding sites, these KCNQ2 structures not only reveal different ligand recognition and activation mechanisms, but also provide a structural basis for drug optimization and design.

Cell Research (2021) 31:52-61; https://doi.org/10.1038/s41422-020-00410-8

\section{INTRODUCTION}

KCNQ2 and its homolog KCNQ3 form homo- and heterotetrameric ion channels on the neuron plasma membrane and are responsible for the $M$-current, which prevents the neurons from over-excitations. ${ }^{1,2}$ Mutations in the KCNQ2 or KCNQ3 gene usually induce a reduced $\mathrm{M}$-current, which leads to excessive excitability of neurons, and the occurrence of seizures. ${ }^{3,4}$ One type of epilepsy, the benign familial neonatal seizures (BFNS or BFNE) are mainly attributed to autosomal dominant mutations in the KCNQ2 gene. Mutations in KCNQ2 gene also result in epileptic encephalopathy. ${ }^{5-7}$ These make KCNQ2 an important drug target to treat epilepsy.

In addition, modulation of KCNQ2 is a potential therapeutic strategy for the treatment of many other diseases such as pain, Parkinson's disease, ischemia, schizophrenia, and smooth muscle disorders, which are characterized by hyper-excitation of neurons..$^{8-11}$ In particular, the expression of KCNQ2 in sensory and central neurons is involved in nociceptive pathways. Suppression of $M$-current in dorsal root ganglion neurons contributes to the development of bone cancer pain, osteoarthritic pain and neuropathic pain, ${ }^{12-14}$ whereas activation of KCNQ2 channel relieves neuropathic pain and fibromyalgia in animal models. ${ }^{15-17}$

KCNQ2 channel can be regulated by endogenous molecules, such as calmodulin (CaM) and phosphatidylinositol 4,5-bisphosphate $\left(\mathrm{PI}(4,5) \mathrm{P}_{2}\right){ }^{18-21}$ Besides, a list of synthetic compounds can activate KCNQ2 by directly binding to the channel. Retigabine (RTG, or ezogabine), specifically targeting KCNQ channels, ${ }^{22,23}$ is the first approved antiepileptic drug that acts by promoting activation of potassium channels. RTG was used as an adjunctive treatment for refractory partial onset seizures in adults since Jun 2011. ${ }^{24}$ As a structural analog of RTG, the marketed non-opioid analgesic flupirtine can also activate KCNQ2. ${ }^{17}$ Flupirtine has been used for the treatment of acute and chronic pain for decades in Europe. $^{25}$ Another compound ICA-27243 was discovered to selectively activate KCNQ2/3 channel at a site different from RTG in vitro, and exhibit anticonvulsant activity in the mouse epilepsy model. ${ }^{26,27}$ However, both RTG and flupirtine cause a series of side effects due to their poor target selectivity. For instance, clinical trials with RTG have found retinal pigmentation and discoloration of skin after prolonged use. ${ }^{28,29}$ Determining structures of KCNQ2 in complex with its modulators would help to develop KCNQselective activators and overcome the side effects of current analgesic and antiepileptic drugs.

Recently, structures of cardiac KCNQ1-CaM complex from Xenopus laevis and human (Homo sapiens, Hs) reveal the overall architecture and gating mechanism of KCNQ1, ${ }^{30,31}$ yet structures of KCNQ in complex with small-molecule activators are not available. To reveal the ligand activation mechanisms of KCNQ2 and to facilitate antiepileptic and analgesic drug development, here we present structural studies of the human KCNQ2 channel.

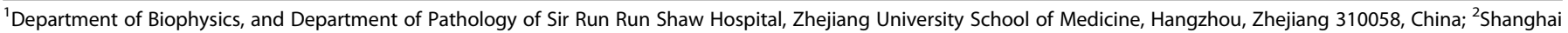

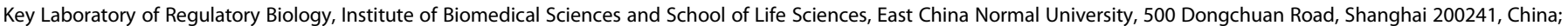

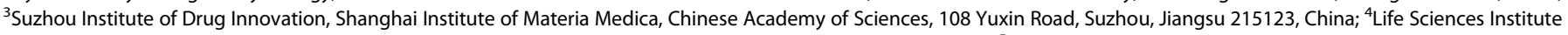

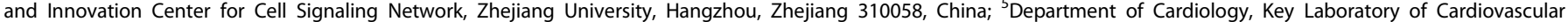

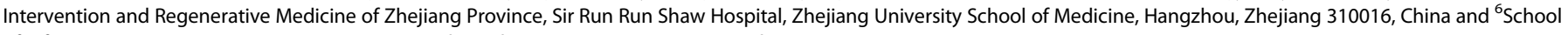
of Life Sciences, Tianjin University, 92 Weijin Road, Nankai District, Tianjin 300072, China

Correspondence: Huaiyu Yang (hyyang@bio.ecnu.edu.cn) or Jiangtao Guo (jiangtaoguo@zju.edu.cn)

These authors contributed equally: Xiaoxiao Li, Qiansen Zhang, Peipei Guo
}

Received: 31 March 2020 Accepted: 14 August 2020

Published online: 3 September 2020 

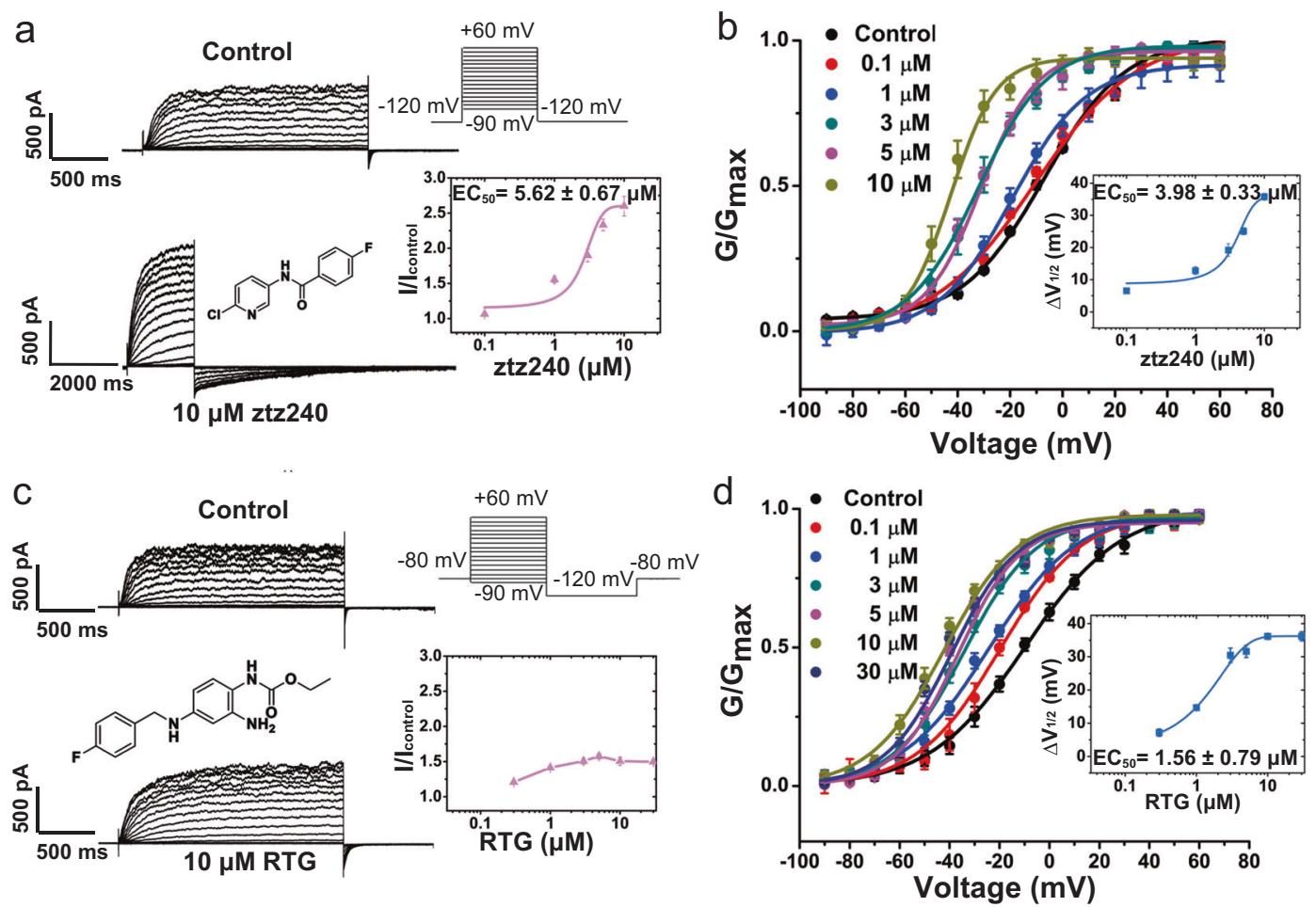

Fig. 1 ztz240 and RTG activation on KCNQ2. a The representative current traces of KCNQ2, before (upper traces) and after (lower traces) application of $10 \mu \mathrm{M}$ ztz240. The inset (left) shows the chemical structure of ztz240. The inset (right) shows the dose-response curve of ztz240 effects on outward current of KCNQ2 at $+50 \mathrm{mV}$, where the ratio of outward current amplitude in the presence of compound (I) versus that in the absence of compound ( $\left.\mathrm{I}_{\text {control }}\right)$ was plotted against agonist compound concentration. $\mathbf{b}$ Voltage activation curves for KCNQ2 generated in the absence and presence of $z t z 240$. The inset shows the concentration-response curve for KCNQ2 channel. $\Delta V_{1 / 2}=V_{1 / 2}$ in control $-V_{1 / 2}$ in the presence of ztz240. c The representative current traces of KCNQ2, before (upper traces) and after (lower traces) application of $10 \mu \mathrm{M}$ RTG. The inset (left) shows the chemical structure of RTG. The inset (right) shows the dose-response curve of RTG effects on outward current of KCNQ2 channel at $+50 \mathrm{mV}$. d Voltage activation curves for KCNQ2 generated in the absence and presence of RTG. The inset shows the concentration-response curve for KCNQ2 channel.

\section{RESULTS}

Functional characterization of activators on human KCNQ2

Two KCNQ2 activators, namely RTG and ztz240, were used in this study. RTG is a classical KCNQ activator, whereas ztz240 is an analog of ICA-27243 and displays a different potentiation activity. ${ }^{32}$ To study the effects of these compounds on KCNQ2 gating, current amplitude-dependent activation $\left(\mathrm{I} / \mathrm{I}_{\text {control }}\right)$ and voltage-dependent activation (G-V) were examined by using whole-cell patch-clamp electrophysiological tests in Chinese hamster ovary (CHO)K1 cells overexpressing KCNQ2 (Fig. 1). Consistent with previous reports, $^{32}$ our electrophysiological results indicate that ztz240 increases outward current amplitude in a dose-dependent manner with a half-maximal value ( $\left.E C_{50}\right)$ of $5.62 \pm 0.67 \mu M(n \geq 5)$ (Fig. 1a). Meanwhile, ztz240 enhances the voltage sensitivity of KCNQ2 by left shifting the G-V curve (Fig. 1b). Along with the increase of ztz240 concentration, the half-activation voltage $\left(V_{1 / 2}\right)$ of $G-V$ curve shifts from $-13.7 \pm 0.50(1 \mu \mathrm{M})$ to $-42.64 \pm 0.93(10 \mu \mathrm{M})(\mathrm{n} \geq 5)$ (Fig. 1b), with an $E C_{50}$ of $3.98 \pm 0.33 \mu \mathrm{M}(n \geq 5)$ fitted from data points of $V_{1 / 2}$ shifts (Fig. 1b). RTG has little effect on the maximal current amplitude (Fig. 1c). Instead, it potentiates KCNQ2 activation mainly by shifting the $\mathrm{V}_{1 / 2}$ of $\mathrm{G}-\mathrm{V}$ curve towards hyperpolarization with an $\mathrm{EC}_{50}$ value of $1.56 \pm 0.79 \mu \mathrm{M}(\mathrm{n} \geq 5)$ (Fig. 1d). Similar activation effect has been observed in previous studies. ${ }^{22,23}$ These results implicate that RTG and ztz240 may have different activation mechanisms on KCNQ2 gating.

Structure determination and overall structures

To prepare the sample for single-particle cryo-electron microscopy (cryo-EM) analysis, a construct covering residues 64-674 of human KCNQ2 was selected, which is of higher purity, homogeneity and yield in comparison with the full-length one. This truncated KCNQ2 exhibits biophysical and pharmacological properties similar to the wild type. In patch clamp experiments, the traces of truncated human KCNQ2 and full-length KCNQ2 were typical (Supplementary information, Fig. S1a). RTG and ztz240 increase the outward current amplitude of $\mathrm{CHO}-\mathrm{K} 1$ cells expressing fulllength and truncated KCNQ2 to the same extent (Supplementary information, Fig. S1b, g). To further test the pharmacological properties of the truncated construct, we examined the voltagedependent characteristic. $5 \mu \mathrm{M}$ RTG and ztz240 left shifted the G-V curve of full-length KCNQ2, with the $\Delta \mathrm{V}_{1 / 2}$ of $31.62 \pm 1.84 \mathrm{mV}$ for RTG (Supplementary information, Fig. S1c) and $25.07 \pm 1.12 \mathrm{mV}$ for ztz240 (Supplementary information, Fig. S1e). For the truncated KCNQ2, the $\Delta \mathrm{V}_{1 / 2}$ is $28.79 \pm 1.41 \mathrm{mV}$ for RTG (Supplementary information, Fig. S1d) and $23.43 \pm 1.52 \mathrm{mV}$ for ztz240 (Supplementary information, Fig. S1f). The statistical results showed that the pharmacological properties of the truncated construct are similar to those of the full-length KCNQ2 (Supplementary information, Fig. S1g, h).

KCNQ2 structures were determined in apo state and in the presence of ztz240 or RTG $+\mathrm{PI}(4,5) \mathrm{P}_{2}$ at $3.1-3.3 \AA$ resolutions, with well-resolved transmembrane domains (TMDs) but poor densities at the cytosolic domain (CD) (Supplementary information, Figs. S2-S4, Table S1). For each condition, during the 3D classifications, we observed one class with stronger densities at the cytosolic region and the crystal structure of KCNQ2/3 proximal $\mathrm{C}$-terminal domain in complex with $\mathrm{CaM}^{33}$ can fit well into the map (Supplementary information, Figs. S2i, S3g, and S4g). Mass spectrometry (MS) analysis confirmed that a fraction of endogenous CaM was co-purified with KCNQ2 (Supplementary information, 

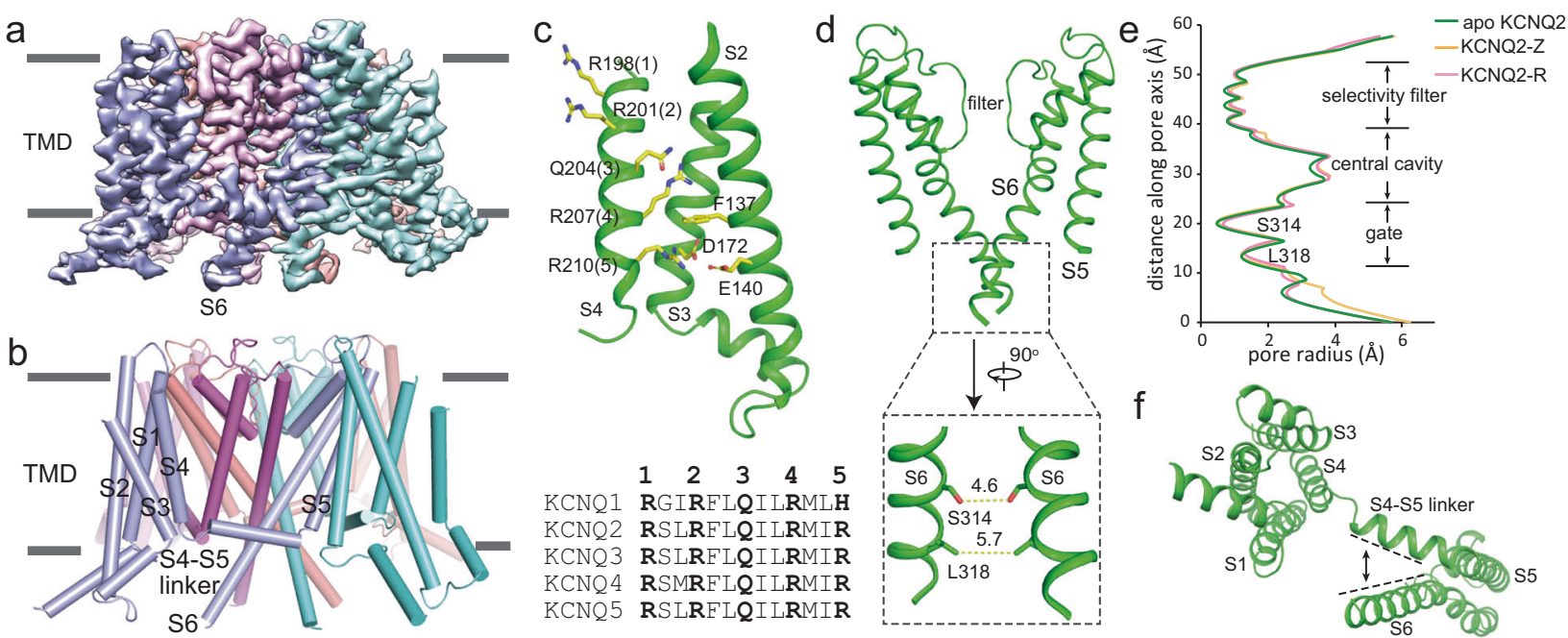

Fig. 2 The apo-state structure of KCNQ2. $\mathbf{a}$, b The cryo-EM map and cartoon model of KCNQ2 with each subunit colored individually. $\mathbf{c}$ The VSD structure of KCNQ2 and sequence alignment of S4 in KCNQ family. For clarity purpose, S1 is not shown. Side chains of positively charged (or polar) residues in S4 and residues forming gating charge transfer center are displayed as sticks. $\mathbf{d}$ The ion conduction pore composed of a pair of diagonal S5-S6, and a zoom-in view of the activation gate. Numbers are diagonal distances (in $\AA$ ) of the constriction points. e The pore radius along the central axis of KCNQ2 in apo, ztz240-bound (KCNQ2-Z) and RTG-bound (KCNQ2-R) states. $\mathbf{f}$ The loose interactions between S4-S5 linker and S6 viewed from intracellular side (also see Supplementary information, Fig. S6b).

Fig. S5d). The three KCNQ2-CaM complex structures were refined to $3.6-3.9 \AA$ resolutions and they are essentially identical to the corresponding KCNQ2 structures in the TMDs. We will focus on the higher-resolution structures of KCNQ2 with TMDs for the analysis of ligand recognition.

The KCNQ2 adopts a canonical domain-swapping conformation, with S5-S6 constituting a central ion conduction pore and S1-S4 forming four voltage-sensing domains (VSDs) at the periphery (Fig. 2a, b). In the VSD, the side chain of Arg210 (R5) points to the gating charge transfer center which is formed by Phe137 and Glu140 in S2, and Asp172 in S3. ${ }^{34}$ Four positively charged (or polar) residues in $\mathrm{S} 4$ are above the conserved aromatic residue Phe137 in S2 (Fig. 2c). Thus, the VSD is in an activated state, similar to the activated conformation of the VSD in HsKCNQ1-CaM (Supplementary information, Fig. S6a). ${ }^{31}$ The apo KCNQ2 is in the closed state, with the shortest diagonal atom-to-atom distance of 4-6 $\AA$ at the activation gate (Fig. $2 d$, e). The closed pore and activated VSD is likely caused by loss of $\mathrm{PI}(4,5) \mathrm{P}_{2}$, resulting in the interruption of tight interactions between S4-S5 linker and S6 (Fig. 2f; Supplementary information, Fig. S6b). Although KCNQ2 shares a similar overall architecture with HsKCNQ1 in the TMD, differences involving all the six transmembrane segments have been observed between two structures, with a root mean square deviation of $3.2 \AA$ over $952 \mathrm{Ca}$ atoms for a tetrameric channel (Supplementary information, Fig. S6c).

\section{Structural basis for ztz240 recognition}

In the ztz240-bound structure (KCNQ2-Z), strong isolated densities are observed in the side cleft between S3 and S4, allowing us to model one ztz240 molecule in each VSD (Fig. 3a, b). A zoom-in analysis shows that ztz240 sits in the gating charge transfer center and forms direct interactions with the conserved residues Phe137, Asp172, and two gating charge residues Arg207 (R3) and Arg210 (R4) in S4 (Fig. 3c). While ztz240 is stabilized mainly by hydrophobic interactions (Fig. 3b), the side chain of Arg210 forms a hydrogen bond with ztz240 (Fig. 3c). Thus, by accurately defining ztz240-binding site, here we provide direct evidence to support previous discoveries that ztz240 and ICA-27243 activate KCNQ2 by binding to the VSD. ${ }^{27,35}$

To confirm the ztz240-binding site, we then measured the electrophysiology of wild type and mutants of KCNQ2 in the presence of $5 \mu \mathrm{M}$ ztz240. Among five mutation sites around the ztz240-binding pocket that were tested, F137A, D172A and R210Q significantly decreased the potentiation activity of ztz240 by reducing the increase of outward current amplitude ratio and preventing or attenuating the left-shift of the G-V curve $\left(\Delta \mathrm{V}_{1 / 2}\right)$ (Fig. 3d; Supplementary information, Fig. S7, Table S2). Mutation R207Q attenuated the left-shift of G-V curve but did not change the outward current amplitude. In contrast, mutation I171A decreased the outward current amplitude but did not affect the left-shift of G-V curve caused by ztz240 binding (Fig. 3d; Supplementary information, Fig. S7). Collectively, these data confirmed the binding site of ztz240 in the VSD.

ztz240 shows subtype selectivity among the KCNQ family. Neither KCNQ1 nor KCNQ3 displays detectable sensitivity to ztz240, while ztz240 potentiates KCNQ4 and KCNQ5 more potently. ${ }^{32}$ By comparing the VSD structures of KCNQ1 and KCNQ2, we observed that KCNQ1 does not possess a similar ztz240-binding pocket. In KCNQ2, residues Ile171 and Ile209 are located on each side of the ztz240-binding pocket and hold the compound by hydrophobic interaction, with a distance of $\sim 11 \AA$ between $\mathrm{Ca}$ atoms of the two residues (Supplementary information, Fig. S8a). However, in apo KCNQ1, the distance between $\mathrm{Ca}$ atoms of the equivalent residues is $7.8 \AA$, and their side chains further block the access of ztz240 to the center of the VSD (Supplementary information, Fig. S8a). Compared with KCNQ4 or KCNQ5, KCNQ1 and KCNQ3 share lower sequence similarity with KCNQ2. Residues involved in the interactions with ztz240 are less conserved in KCNQ1 or KCNQ3 (Supplementary information, Fig. S8b), which may explain the low sensitivity of KCNQ1 and KCNQ3 to ztz240.

The KCNQ2-Z possesses an activated VSD and a closed pore (Figs. $2 \mathrm{e}$ and $3 \mathrm{e}-\mathrm{g}$ ). While the pore domain remains unchanged, ztz240 binding induces multiple local conformational changes in the VSD. First, S2, S3 and the major part of S1, shift by $2-5 \AA$ relative to those in apo state (Fig. 3e). These conformational changes seem necessary to provide a deep hydrophobic pocket for ztz240 to bind (Fig. 3b). Second, around the hinge between S4 and S4-S5 linker, S4 rotates close to the pore domain, strengthening hydrophobic interactions between S4 and S5 of its neighboring subunit (Fig. 3f). Third, after rotation, S4 is in an even "up" conformation, with $3-4 \AA$ shifts for the first three 

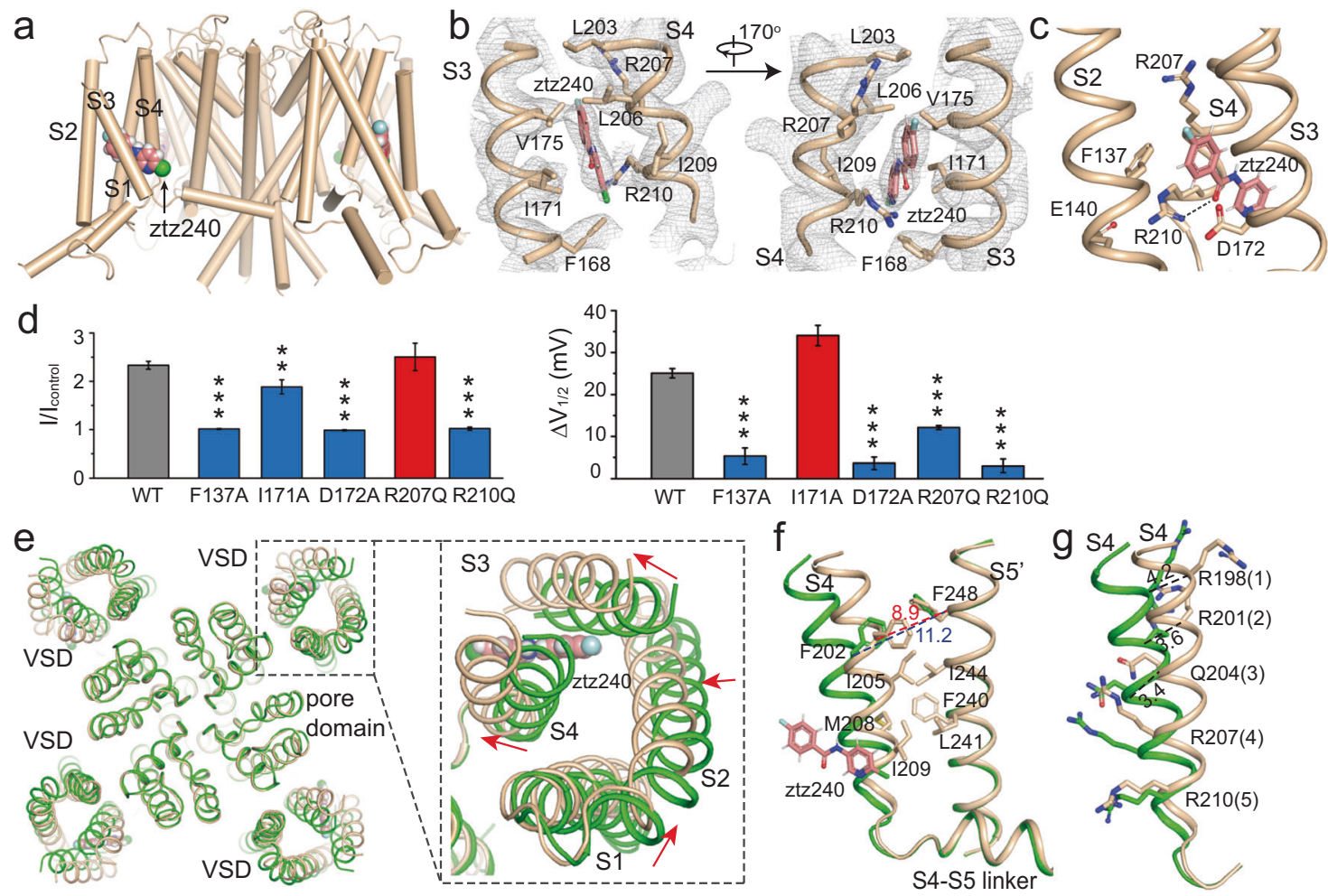

Fig. 3 The ztz240-bound structure of KCNQ2 (KCNQ2-Z). a Localization of ztz240 in KCNQ2. b Local electron densities at the ztz240-binding site at the contour level of $4.5 \sigma$. c ztz240 sits in the gating charge transfer center. The dash line indicates the hydrogen bond between ztz240 and Arg210. d Effects of $5 \mu \mathrm{M}$ ztz240 on outward current amplitude (left columns, the test potential is $+50 \mathrm{mV}$ ) and on $\mathrm{V}_{1 / 2}$ (right columns) of KCNQ2 mutants $\left(n \geq 5,{ }^{* *} P \leq 0.005,{ }^{* * *} P \leq 0.0005\right) . \Delta \mathrm{V}_{1 / 2}$ indicates the change of $\mathrm{V}_{1 / 2}$ after application of $z t z 240 . \Delta \mathrm{V}_{1 / 2}=\mathrm{V}_{1 / 2}$ in control $-\mathrm{V}_{1 / 2}$ in the presence of $5 \mu \mathrm{M}$ ztz240. Data are presented as means \pm SEM. e Shifts of VSD upon ztz240 binding. The apo-state structure (green) and ztz240-bound structure (wheat color) are aligned at the pore domain (Ser229-Lys327) which remains almost unchanged upon ztz240 binding. Red arrows indicate the shift directions. f ztz240 strengthens hydrophobic interactions between S4 and S5. Numbers shows distances (in $\AA$ ) between $\mathrm{C} \alpha$ atoms of Phe202 in S4 and Phe248 in S5' from the neighboring subunit before (blue) and after (red) ztz240 binding. $\mathbf{g}$ Structural superimposition of S4 in apo state (green) and ztz240-bound state (wheat). Numbers show distances (in $\AA$ ) between C $\alpha$ atoms of R1-3 from two S4 structures.

charges (R1-R3, Fig. 3g). Therefore, ztz240 acts as a wedge, which inserts into the hydrophobic pocket between S3 and S4 and stabilizes the activated conformation of S4. Based on these observations, we propose that ztz240 may activate KCNQ2 by holding the VSD in the activated conformation.

\section{Structural basis for RTG recognition}

In the RTG-bound KCNQ2-R structure, bulk densities arising from the RTG molecule are observed in a hydrophobic pocket formed by S5, pore helix, and S6 from the neighboring subunit at the inter-subunit interface in the pore domain (Fig. 4a, b). The shape of the densities accurately defines the orientation and conformation of the RTG molecule. RTG binds to KCNQ2 mainly by hydrogen bonds with the side chain of Trp236, Ser303, and the main chain carbonyls of Leu299, Phe305, as well as the hydrophobic interactions with residues Trp236, Phe240, Leu243, Leu272, Leu299, Phe304 and Phe305 (Fig. 4c, d). Although PI $(4,5)$ $\mathrm{P}_{2}$ was added in the sample, no densities corresponding to $\mathrm{PI}(4,5)$ $\mathrm{P}_{2}$ molecules are observed in the EM map, probably due to its dynamic interactions with KCNQ2. ${ }^{21}$

To verify the RTG-binding site, we measured the electrophysiology of wild type and four KCNQ2 mutants in the presence of $5 \mu \mathrm{M}$ RTG. RTG activates KCNQ2 channel mainly by inducing a hyperpolarizing shift of the G-V curve (Fig. 1d). Therefore, we examined the effects of these mutations on left-shift of G-V curves in the presence or absence of RTG. All of the four mutations we tested, W236A, F240A, L299A and S303A, decreased the potentiation activity of RTG on KCNQ2 by preventing or attenuating the left-shift of the G-V curves $\left(\Delta \mathrm{V}_{1 / 2}\right)$ (Fig. 4e; Supplementary information, Fig. S9). Consistently, multiple previous mutagenesis studies on KCNQ2 and other KCNQ channels supported this RTGbinding site. ${ }^{36-38}$ Specifically, the prediction that Trp236 forms a direct hydrogen bond with RTG via its indole ring is validated by our structure data. ${ }^{39}$

Sequence alignment shows that residues lining the RTG-binding pocket are highly conserved across KCNQ family except KCNQ1 (Supplementary information, Fig. S10a). Among the nine residues that directly interact with RTG in KCNQ2, four are replaced in KCNQ1, including the important Trp236 in S5. In KCNQ2, Trp236 in S5, Leu299 and Ile300 in S6 from the neighboring subunit participate in building a deep hydrophobic pocket for RTG to bind, whereas in KCNQ1, the equivalent residues are Leu266, Val334 and Phe335. In KCNQ1, Leu266 and Phe355 are closer to each other and their side chains form strong hydrophobic interactions, preventing RTG to enter the inter-subunit interface in the TMD (Supplementary information, Fig. S10b). Therefore, it is not surprising that RTG activates other KCNQ channels but not KCNQ1.

The RTG-bound structure KCNQ2-R also holds an activated VSD and a closed pore (Figs. 2e and $4 \mathrm{f}-\mathrm{g}$ ). RTG binding causes conformational changes mainly in pore domain, with a clockwise rotation of upper halves of S5, S6 and the pore helix viewed from the extracellular side (Fig. 4f). RTG inserts into the interface between $\mathrm{S} 5$ and $\mathrm{S} 6$ from its neighboring subunit and pushes them away from each other by almost 1-2 $\AA$. The side chain of Trp236 moves outwards by $\sim 2 \AA$ to empty space for RTG (Fig. 4g). Although the VSD in KCNQ2-R structure remains almost 

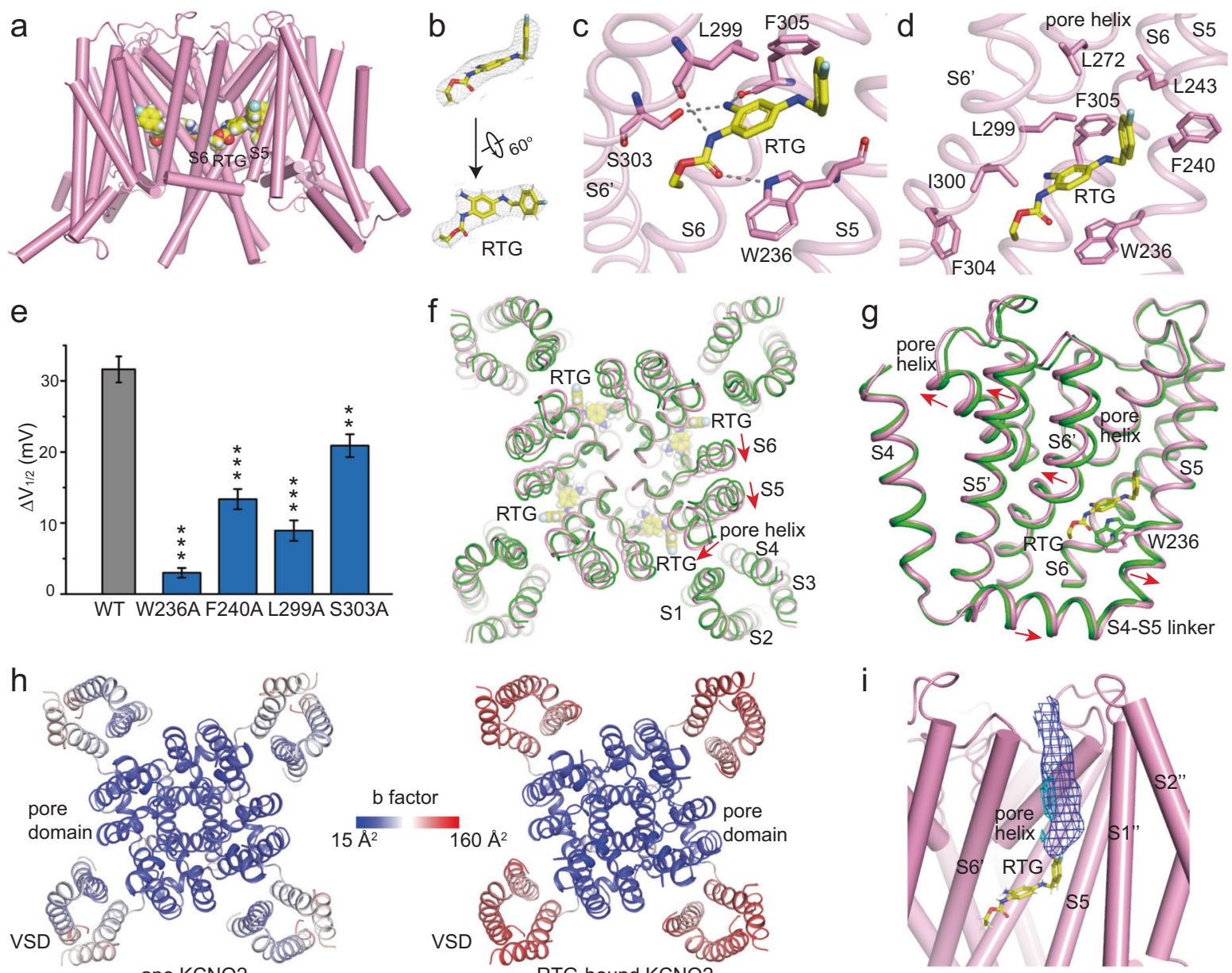

Fig. 4 The RTG-bound structure of KCNQ2 (KCNQ2-R). a Localization of RTG in KCNQ2. b Electron densities of RTG at the contour level of $5 \sigma$. c Hydrogen bonds (dash line) between RTG and its surrounding residues. d Hydrophobic interactions between RTG and its surrounding residues. e Effects of $5 \mu \mathrm{M}$ RTG on $V_{1 / 2}$ of KCNQ2 mutants $\left(n \geq 5,{ }^{* *} P \leq 0.005\right.$, $\left.{ }^{* * *} P \leq 0.0005\right) . \Delta V_{1 / 2}$ indicates the change of $V_{1 / 2}$ after application of RTG. $\Delta \mathrm{V}_{1 / 2}=\mathrm{V}_{1 / 2}$ in control $-\mathrm{V}_{1 / 2}$ in the presence of $5 \mu \mathrm{M}$ RTG. Data are presented as means \pm SEM. $f$ RTG induced conformational changes at the upper half of the pore domain. The apo (green) and RTG-bound (pink) structures are aligned at TMDs using secondary structure matching (SSM) method. Red arrows indicate the motion directions. The apo-state structure is show in green and the RTG-bound structure in pink. g Shifts of S1, S2 and S3 upon RTG binding. $\mathbf{h}$ Structures of the apo and RTG-bound KCNQ2 colored by b factors. i Electron densities corresponding to putative lipids disappear upon RTG binding. Densities from the map of apo state are shown in blue mesh and from the RTG-bound map are in cyan ( $5 \sigma$ level).

unchanged, the high B-factor indicates that it displays increased mobility upon RTG binding (Fig. 4h). The disturbance of the VSD may also be promoted by loss of lipids in the groove formed by S1, S5 and pore helix. RTG occupies the position of the hydrophobic tail of the putative lipid and seems to push it away from the groove (Fig. 4i). RTG here acts as an allosteric modulator, binding to the pore domain but changing the channel's voltage dependence.

The KCNQ2-CaM complex structures

In the apo KCNQ2-CaM structure, following S6, three cytosolic helices are observed, namely $\mathrm{HA}, \mathrm{HB}$ and $\mathrm{HC}$. $\mathrm{HA}$ and $\mathrm{HB}$ form a hairpin and are tightly wrapped by one $\mathrm{CaM}$, whereas the four HCs form a coiled coil along the central axis of the tetrameric channel (Fig. 5a). In comparison with HsKCNQ1-CaM, a large displacement is observed in the $C D$ and $C a M{ }^{31}$ First, structural and sequence alignments show that the short loop connecting $\mathrm{S} 6$ and HA shifts backward by 3-4 residues in KCNQ2-CaM (Fig. 5b). Second, around the hinge between $\mathrm{HA}$ and $\mathrm{S} 6, \mathrm{HA}$ and $\mathrm{HB}$ in KCNQ2-CaM rotate by $\sim 45^{\circ}$ relative to those in HsKCNQ1 (Fig. 5b). Consequently, the CaM surrounding the $\mathrm{HA}$ and $\mathrm{HB}$ helices shifts by about 10-15 $\AA$, relative to that in HsKCNQ1-CaM (Fig. 5c). Third, in the apo
KCNQ2-CaM, the four HA-HB-CaM domains are separated from each other, whereas in KCNQ1-CaM, they constitute a stable cytosolic square ring with direct interactions between $\mathrm{N}$-lobe of $\mathrm{CaM}$ and the HA from its neighboring subunit (Fig. $5 \mathrm{C}$ ). The different structural features of the HA-HB-CaM may account for the different calcium sensitivities and gating properties between KCNQ1 and KCNQ2. ${ }^{40}$

The overall architecture of ztz240-bound KCNQ2-CaM structure (KCNQ2-CaM-Z) is similar to the apo-state structure (Supplementary information, Fig. S11a). By contrast, in the RTG-bound KCNQ2CaM structure (KCNQ2-CaM-R), large structural rearrangements occur in CD and CaM. In the apo KCNQ2-CaM, S6 and HA form a $\sim 120^{\circ}$ angle, connected by a short loop containing residues 330-332, and HB sits on the right side of HA (Supplementary information, Fig. S11b). In KCNQ2-CaM-R, S6 and HA become one jointed bending helix, and HA-HB-CaM undergoes almost $180^{\circ}$ rotation, with HB moving to the left side of HA (Supplementary information, Fig. S11b). As the RTG-bound structure was determined in the presence of both RTG and $\mathrm{PI}(4,5) \mathrm{P}_{2}$, to distinguish which one induces such large conformational changes, we also determined a 6-7 $\AA$ resolution structures of KCNQ2-CaM in the presence of only RTG. The apo KCNQ2-CaM can fit well into 

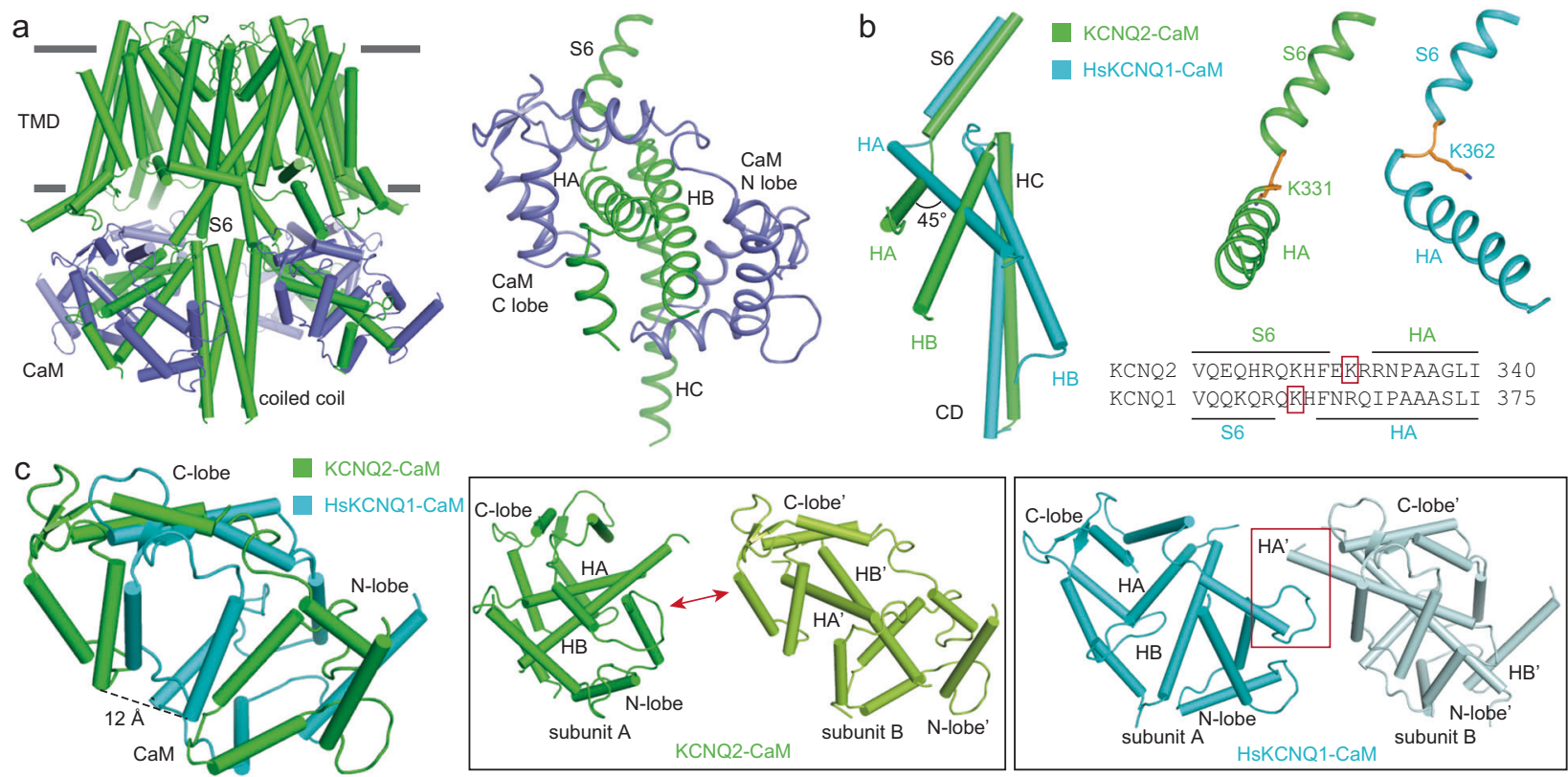

Fig. 5 The structure of KCNQ2-CaM complex. a The apo KCNQ2-CaM structure and a zoom-in view of the CD (green) and CaM (blue). b Structural alignment of KCNQ2-CaM (green) and HsKCNQ1-CaM (cyan, PDB: 6UZZ) at CD, and the side-by-side comparison of the S6-HA structures in KCNQ2-CaM and HsKCNQ1-CaM. c Structural comparisons of CaM in KCNQ2-CaM (green) and HsKCNQ1-CaM (cyan). The two adjacent HA-HB-CaM domains are separated from each other in KCNQ2-CaM (middle), whereas they form direct interactions in HsKCNQ1-CaM, as highlighted by the red box (right).

the map with only RTG, whereas the KCNQ2-CaM-R structure cannot (Supplementary information, Fig. S11c), suggesting that the structure rearrangements in $C D$ and CaM of KCNQ2-CaM is induced by $\mathrm{PI}(4,5) \mathrm{P}_{2}$. However, in KCNQ2-CaM-R structure, the gate remains closed and $\mathrm{PI}(4,5) \mathrm{P}_{2}$ was not unambiguously assigned, hindering us from providing an activation mechanism. The structure rearrangements in the HA-HB-CaM of KCNQ2-CaM resembles those observed in HsKCNQ1-CaM-KCNE3 complex, implicating a conserved activation mechanism among KCNQ family. ${ }^{31}$ In HsKCNQ1-CaM-KCNE3 structure, $\mathrm{PI}(4,5) \mathrm{P}_{2}$ binds at the cleft formed by S3, S4 and S4-S5 linker. By interacting with positively charged side chains contributed by S0, the S2-S3 loop, and the $\mathrm{S} 4-\mathrm{S} 5$ linker, $\mathrm{PI}(4,5) \mathrm{P}_{2}$ induces the structure rearrangements in $\mathrm{HA}-\mathrm{HB}-\mathrm{CaM}$ and activates the channel. $\mathrm{PI}(4,5) \mathrm{P}_{2}$ may play a similar role in KCNQ2-CaM complex.

\section{DISCUSSIONS}

The ztz240-binding site in VSD

Modulation of VSD is an effective strategy for discovering selective drug molecules. It has been demonstrated that modifiers can regulate voltage-gated potassium (Kv) channels, sodium (Nav) channels and proton (Hv) channels by binding to the upper vestibule of the VSD, ${ }^{41-47}$ yet the discovery of modifiers binding to the VSD from the side cleft has been rarely reported. In KCNQ2, we identify the ztz240-binding site in side cleft between S3 and S4 by both cryo-EM and electrophysiology analyses. This site, to our surprise, differs from other ztz240 analogs' binding sites derived from mutagenesis studies. For example, both ICA-27243 and ICA069673 (ICA73) are predicted to bind at the extracellular side of KCNQ2 VSD. ${ }^{27,48}$ Considering their high structural similarity, these two compounds are very likely to share the same site with ztz240. Residues located at the extracellular side of VSD may regulate the compounds' sensitivity by allosteric effects. In fact, we do observe conformational changes at the extracellular side of the VSD upon ztz240 binding (Fig. 3e-g). NH29 is a new KCNQ2 opener that can produce a hyperpolarizing shift of the activation curve and slow both activation and deactivation kinetics. It was also suggested that $\mathrm{NH} 29$ binds to the external groove of VSD formed by the interface of helices S1, S2, and S4. ${ }^{41}$ Due to the differences in the structures of $\mathrm{NH} 29$ and ztz240, it is possible that they activate KCNQ2 by binding to different sites in the VSD.

This ztz240-binding site is different from other ligand-binding sites in the VSDs of voltage-gated and ligand-gated ion channels (Supplementary information, Fig. S12a). In Nav1.7, the antagonist GX-936 sits deeply within the extracellular cleft of the VSD4 and traps the VSD in the activated state (Supplementary information, Fig. S12a). ${ }^{43}$ In TRPM8, two hydrophobic antagonists, namely AMTB and TC-I 2014, were captured in the lower half of the VSD (Supplementary information, Fig. S12a). ${ }^{49}$ Furthermore, in TRPC5, the inhibitor clemizole was identified at the similar pocket of VSD. ${ }^{50}$ The latter three ligands bind to the cytosolic facing pocket in the VSD, remarkably different from the membrane-embedded cleft between S3 and S4 where ztz240 inserts.

The identification of the ztz240-binding site in KCNQ2 will provide assistance in the design of KCNQ-selective agonists, taking account of the high sequence conservation among KCNQ2, 4 and 5 (Supplementary information, Fig. S8b). Since the ztz240-binding site is located in the middle of the membrane layer, small molecules may need to penetrate into the cell membrane phase first and then access the druggable binding site, rather than from the extracellular or intracellular side of the membrane. Small molecules or drugs that could easily access the phospholipid bilayers may be more conducive to binding to the ztz240-binding pocket.

The RTG-binding site in the pore domain

While the ztz240-binding site is newly identified, the RTG-binding pocket is a classical drug binding site in the pore domain of ion channels. In Cav1.1 structure, the binding pocket of agonist Bay $\mathrm{K}$ 8644 and antagonist nifedipine is similar to that of RTG in KCNQ2 (Supplementary information, Fig. S12b). ${ }^{51}$ Bay K 8644 increases the current densities and left shifts the G-V curve of $\mathrm{Ca}_{\mathrm{v}} 1.1$, similar to the effect of RTG on KCNQ2. In TRPC5, the inhibitor HC-070 sits at a similar hydrophobic pocket built by $\mathrm{S} 5$, pore helix from one subunit and $\mathrm{S} 6$ of the adjacent subunit. ${ }^{50}$ These suggest that the RTG-binding site is a general ligand-binding site in ion channels. 
Potential activation mechanisms

ztz240 and RTG activate KCNQ2 by different mechanisms. ztz240 increases the outward current and enhances the voltage sensitivity, whereas RTG mainly modifies the voltage activation. Although both KCNQ2-Z and KCNQ2-R structures were in the closed states, by analyzing structural and electrophysiology data, we can propose their potential activation mechanisms. By binding to the activated VSD, ztz240 likely reduces the energy barrier from resting to activated VSD, and therefore increases the channel's voltage sensitivity. Upon ztz240 binding, the VSD rotates close to the pore domain and strengthens their coupling. As single KCNQ2 channel has a low mean maximal open probability $\left(P_{o}\right){ }^{52}$ ztz240 probably elevates outward current by increasing the mean maximal $P_{O}\left(P_{O_{-} \text {max }}\right)$ of KCNQ2. Noise analysis of the whole-cell recordings shows that ztz240 enhances KCNQ2 current amplitude by increasing $P_{o \text { max }}$ but not the single channel conductance (Supplementary information, Fig. S13). RTG binds to the pore domain but changes the channel's voltage dependence. It induces local conformational changes at the upper half of the pore domain, which may couple the conformational changes of the lower activation gate when the channel opens. In addition, as the VSD displays increased mobility after RTG binding, under physiological condition RTG presumably also reduces the energy required for the transition of VSD from resting to activated state.

\section{Insights into drug development}

RTG causes serious side effects mainly due to its poor selectivity. Therefore, structural modification of RTG to increase the activity and selectivity of analogs has been carried out. In the KCNQ2-R structure, the $-\mathrm{F}$ group at the benzylamine moiety of RTG is in a hydrophobic cavity, and the modification of $-\mathrm{F}$ group to a larger group to increase hydrophobic interactions may help to improve the activity of RTG analogs. In fact, the substitution of $-\mathrm{F}$ group with a larger hydrophobic group, such as $-\mathrm{CF}_{3}$, could improve the activity of RTG analogs on $\mathrm{M}$ channel. ${ }^{53}$ Meanwhile, residue Leu272 in KCNQ2 is near the $-\mathrm{F}$ group and it is not conserved in KCNQ1, KCNQ4 and KCNQ5. Therefore, the modification of $-\mathrm{F}$ group may improve the selectivity of RTG analogs to KCNQ2, KCNQ3 channels. When the $-\mathrm{NH}_{2}$ group was modified to other, the RTG analogs showed reduced activation or inhibitory effect on KCNQ2 channel. ${ }^{54,55}$ This may be because $-\mathrm{NH}_{2}$ group in RTG can form a hydrogen bond network with Ser303 and Phe305, which is very important to stabilize the binding of RTG to KCNQ2 (Fig. 4c). The destruction of this effect may reduce the activation of RTG analogs on KCNQ2 channel.

Compared with $\mathrm{ztz240}$, the $-\mathrm{H}$ group at position 3 of the aromatic moiety is replaced with an $-\mathrm{F}$ group in ICA-27243. While ztz240 shows no selectivity among KCNQ2, KCNQ4 and KCNQ5, ICA-27243 is a KCNQ2/Q3 subtype-selective opener. Thus, the $-\mathrm{F}$ group in the aromatic moiety increases the selectivity. In the KCNQ2-Z structure, there is space above the aromatic moiety of ztz240 to accommodate additional groups. In the future, introducing diverse groups at position 3 of the aromatic moiety will be a strategy to develop more selective activators of KCNQ2.

In summary, the KCNQ2 structures in complex with ztz240 and RTG provide first glimpse of ligand activation mechanism of KCNQ channels. The identification of two activators at different binding sites will facilitate future drug development and optimization, in the context of side effects of current analgesic and antiepileptic drugs.

\section{MATERIALS AND METHODS}

Expression and purification of KCNQ2

The truncated human KCNQ2 gene (NCBI accession NP_004509.2) that encodes the KCNQ2 channel covering residues 64-674 was synthesized and cloned into a pEZT-BM vector with a C-terminal strep-tag. ${ }^{56}$ KCNQ2 was heterologously expressed in HEK293S
$\mathrm{GnTI}^{-}$cells using the BacMam system (Thermo Fisher Scientific). The P3 baculovirus derived from sf9 cells following the standard protocol was used to transfect HEK293S $\mathrm{GnTI}^{-}$cells when the cell density reached $3-3.5 \times 10^{6}$ cells $/ \mathrm{mL}$. For $800 \mathrm{~mL}$ of HEK293S $\mathrm{GnTI}^{-}$cells, $60 \mathrm{~mL} \mathrm{P3}$ virus was added, followed by the addition of $10 \mathrm{mM}$ sodium butyrate to enhance protein expression. Cells were cultured at $37^{\circ} \mathrm{C}$ for $48 \mathrm{~h}$ before they were harvested by centrifugation at $3000 \times g$ for $20 \mathrm{~min}$.

The cells were re-suspended in lysis buffer A ( 20 mM Tris, pH 8.0, and $150 \mathrm{mM} \mathrm{KCl}$ ) supplemented with a protease inhibitor cocktail $(2 \mu \mathrm{g} / \mathrm{mL}$ DNase l, $0.5 \mu \mathrm{g} / \mathrm{mL}$ pepstatin, $2 \mu \mathrm{g} / \mathrm{mL}$ leupeptin and 1 $\mu \mathrm{g} / \mathrm{mL}$ aprotinin, and $0.1 \mathrm{mM}$ PMSF) and homogenized by sonication on ice. The KCNQ2 protein was extracted with $1.25 \%$ $(w / v)$ n-Dodecyl- $\beta$-D-Maltopyranoside (DDM, Anatrace) and $0.25 \%$ $(\mathrm{w} / \mathrm{v})$ cholesteryl hemisuccinate (CHS, Anatrace) by gentle agitation for $3 \mathrm{~h}$ at $4{ }^{\circ} \mathrm{C}$. The supernatant was collected after a 50-min ultra-centrifugation at $46,000 \times g$ and then incubated gently with Strep-Tactin Sepharose resin (IBA) at $4{ }^{\circ} \mathrm{C}$ for $1 \mathrm{~h}$. The collected resin was rinsed with buffer $B$ (buffer $A+0.05 \%(w / v)$ DDM) for 10 column volumes. The detergent was then changed to $0.06 \%$ glyco-diosgenin (GDN) and KCNQ2 was eluted with $10 \mathrm{mM}$ desthiobiotin in buffer C (buffer $A+0.06 \% \mathrm{GDN}$ ). The protein sample was further purified by size-exclusion chromatography with a Superose 6 10/300 GL column (GE Healthcare) equilibrated with buffer $C$. The peak fractions were pooled in a $1.5-\mathrm{mL}$ centrifuge tube for single-particle cryo-EM analyses.

\section{MS analysis}

The KCNQ2 sample was always co-purified with an endogenous protein with a molecular weight of $\sim 10-15 \mathrm{kD}$ on SDS-PAGE gel (Supplementary information, Fig. S5b). To identify this endogenous protein, we did MS analysis. The SDS-PAGE gel was washed for several times with double-distilled water, and the target band was excised carefully and placed into a $1.5-\mathrm{mL}$ centrifuge tube. The short peptides generated through enzymolysis were identified using liquid chromatography-tandem mass spectrometry (LC-MS/ MS) method by Sangon Biotech (Shanghai) Co., Ltd. The MS results indicated that both $\mathrm{CaM}$ and KCNQ2 were included in the sample. The identified peptides of KCNQ2 are mainly distributed in HA and HB helices, which directly form strong interactions with CaM (Supplementary information, Fig. S5c, d). Therefore, this 10-15 kD band on SDS-PAGE gel arises from both CaM and a portion of degraded KCNQ2 that is co-localized with CaM.

Preparation of KCNQ2 in complex with activators RTG and ztz240 were purchased from TargetMol (USA) and Topscience Co. (Shanghai, China), respectively. For the sample without any compound, the peak fractions of size exclusion chromatography were concentrated to $4 \mathrm{mg} / \mathrm{mL}$ directly. For KCNQ2 bound to ztz240 or RTG, $400 \mu \mathrm{L}$ peak fractions were mixed with ztz240 or RTG to a final concentration of $250 \mu \mathrm{M}$. After incubation at $4{ }^{\circ} \mathrm{C}$ for $20 \mathrm{~min}$, the mixture sample was then concentrated to $\sim 3 \mathrm{mg} / \mathrm{mL}$ for cryo-EM sample preparation. For KCNQ2 bound to $\mathrm{RTG}+\mathrm{PI}(4,5) \mathrm{P}_{2}$, peak fractions containing target protein were concentrated to $50 \mu \mathrm{L}$ and incubated with equal volume of $1 \mathrm{mM} \mathrm{PI}(4,5) \mathrm{P}_{2}$ (Avanti) at $4{ }^{\circ} \mathrm{C}$ for $30 \mathrm{~min}$, followed by a further incubation at $4{ }^{\circ} \mathrm{C}$ for $20 \mathrm{~min}$ with the addition of $250 \mu \mathrm{M}$ RTG. The mixture of KCNQ2-PI(4,5) $\mathrm{P}_{2}-\mathrm{RTG}$ was finally concentrated to $\sim 3 \mathrm{mg} / \mathrm{mL}$ for cryo-EM sample preparation.

Cryo-EM data acquisition

The cryo-EM data of KCNQ2 was collected at Center of CryoElectron Microscopy at Zhejiang University. $3 \mu \mathrm{L}$ aliquots of KCNQ2 were placed on glow-discharged holey carbon grids (Quantifoil $\mathrm{Cu}$ R1.2/1.3, 300 mesh), which were blotted for $3 \mathrm{~s}$ under $100 \%$ humidity at $4{ }^{\circ} \mathrm{C}$ before being plunged into liquid ethane using a Mark IV Vitrobot (FEI). The grids were loaded onto a Titan Krios microscope (FEl) operated at $300 \mathrm{kV}$ with a K2 Summit direct 
electron detector (Gatan). SerialEM software was used for automated data collection following standard procedure. A calibrated magnification of 49,310x was used for imaging, yielding a pixel size of $1.014 \AA$ on images. Data were dose-fractionated to 40 frames with a dose rate of $8 \mathrm{e}^{-} / \mathrm{pixel} / \mathrm{s}$ and a total exposure time of $8 \mathrm{~s}$, corresponding to a total dose of $\sim 62 \mathrm{e}^{-} / \AA^{2}$.

Image processing

The motion correction was performed using the MotionCorr2 program, ${ }^{57}$ and the CTF parameters of the micrographs were estimated using the GCTF program. ${ }^{58}$ All subsequent steps of image processing were carried out with RELION. ${ }^{59}$ For the sample without any exogenous compound, 1000 particles were manually picked from the micrographs for $2 D$ classification initially. Several KCNQ2 representative 2D class averages in different orientations were selected as templates for automated particle picking. The auto-picked 2,319,760 particles from the 3936 micrographs were subjected a 2D classification, and a total of 973,011 particles were selected for two rounds of 3D classification using the initial model generated by RELION as the reference. After the second round of 3D classification, 78,605 particles from one 3D class were selected for 3D reconstruction, yielding a $3.6 \AA$ resolution map after $3 \mathrm{D}$ refinement with $\mathrm{C} 4$ symmetry and particle polishing (KCNQ2-CaM). Meanwhile, 205,465 particles from another 3D class were selected and subjected to a third round of $3 D$ classification, and 145,412 particles from one 3D class were selected for 3D reconstruction, yielding a $3.1 \AA$ resolution map after $3 \mathrm{D}$ refinement with $\mathrm{C} 4$ symmetry and particle polishing (KCNQ2) (Supplementary information, Fig. S2). For the samples with activators, data processing was performed following similar procedures (Supplementary information, Figs. S3, S4). The resolutions were estimated by applying a soft mask around the protein density and the gold-standard Fourier shell correlation $(\mathrm{FSC})=0.143$ criterion (Supplementary information, Figs. S2f, S3d, S4d). RELION was used to calculate the local resolution maps. ${ }^{59}$

Model building, refinement, and validation

De novo atomic model building was performed in $\mathrm{Coot}^{60}$ based on the $3.1 \AA$ resolution map of the apo KCNQ2. Amino acid assignment was achieved based on the clearly defined density for bulky residues (Phe, Trp, Tyr, and Arg). Models were refined against summed maps using phenix.real_space_refine, ${ }^{61}$ with secondary structure restraints and non-crystallography symmetry applied. The initial EM density map allowed us to construct a KCNQ2 model containing residues $70-184$ and 195-330. For KCNQ2-CaM, the crystal structure of a chimeric Kv7.2-Kv7.3 proximal C-terminal domain in complex with CaM (PDB: 5J03) and the high-resolution cryo-EM structure of HsKCNQ1 (PDB: $6 U Z Z$ ) were used as references for model building in CD and CaM. The models of apo KCNQ2 and KCNQ2-CaM work as references for building models with activators. The statistics for the models' geometries was generated using MolProbity ${ }^{62}$ (Supplementary information, Table S1). All the structure figures were prepared in PyMol $^{63}$ or Chimera. ${ }^{64}$

\section{Mutagenesis}

The CDNAs of human KCNQ2 were subcloned into the pIRES2EGFP vector. All site-directed mutagenesis were conducted and sequencing confirmed by providers (GENEWIZ, Suzhou, China).

\section{Cell culture and transient transfection}

CHO-K1 cells were grown in 50/50 DMEM/F12 (Gibco, Carlsbad, CA, USA) with $10 \%$ FBS and $2 \mathrm{mM}$ L-glutamine (Gibco, Carlsbad, CA, USA). To express ion channels, cells were split at $24 \mathrm{~h}$ before transfection, plated in $60-\mathrm{mm}$ dishes and transfected with Lipofectamine $3000^{\mathrm{TM}}$ reagent (Invitrogen, Carlsbad, CA, USA), according to the manufacturer's instructions. Electrophysiological recordings were performed between 24 and $48 \mathrm{~h}$ after transfection.

Electrophysiological recording

Whole-cell voltage clamp recording was carried out at room temperature in CHO-K1 cells with the amplifier EPC-10 (HEKA). The electrodes were pulled from borosilicate glass capillaries (World Precision Instruments, Sarasota, FL, USA). When filled with the intracellular solution, the electrodes had resistances of 3-5 M 2 . Pipette solution contained $150 \mathrm{mM} \mathrm{KCl}, 3 \mathrm{mM} \mathrm{MgCl} 2,5 \mathrm{mM}$ EGTA, $10 \mathrm{mM}$ HEPES ( $\mathrm{pH} 7.3$ with $\mathrm{KOH}$ ). During the recording, constant perfusion of extracellular solution was maintained using a gravityfed continuous focal perfusion system. Extracellular solution contained $145 \mathrm{mM} \mathrm{NaCl}, 5 \mathrm{mM} \mathrm{KCl}, 1 \mathrm{mM} \mathrm{CaCl} 2,3 \mathrm{mM} \mathrm{MgCl}_{2}, 10$ mM HEPES (pH 7.4 with $\mathrm{NaOH}$ ). The current signals were filtered at $2 \mathrm{kHz}$ and digitized at $10 \mathrm{kHz}$. Series resistance was compensated by $60 \%-80 \%$. To elicit currents, cells were stimulated by a series of $2000 \mathrm{~ms}$ depolarizing steps from $-90 \mathrm{mV}$ to $+60 \mathrm{mV}$ in $10 \mathrm{mV}$ increments at a holding potential of $-80 \mathrm{mV}$ (testing RTG) or -120 $\mathrm{mV}$ (testing ztz240).

Data and statistical analysis

Patch-clamp data were processed using Clampfit 10.4 (Molecular Devices), and then analyzed in GraphPad Prism 8 (GraphPad Software, San Diego, CA, USA). Voltage-dependent activation curves were fitted with the Boltzmann equation: $G=$ $G_{\min }+\left(G_{\max }-G_{\min }\right) /\left(1+\exp \left(V-V_{1 / 2}\right) / S\right)$, where $G_{\max }$ is the maximum conductance, $G_{\min }$ is the minimum conductance, $V_{1 / 2}$ is the voltage for reaching $50 \%$ of maximum conductance and $S$ is the slope factor. Dose-response curves were fitted with the Hill equation: $E=E_{\max } /\left(1+\left(\mathrm{EC}_{50} / \mathrm{C}\right)^{\mathrm{P}}\right)$, where $\mathrm{EC}_{50}$ is the drug concentration producing half of the maximum response and $\mathrm{P}$ is the Hill coefficient. The activation and deactivation traces were fitted with exponential equations containing one or two components using Clampfit 10.4. Data are presented as means \pm SEM. Significance was estimated using paired two-tailed Student's $t$ test. Noise analysis was applied to estimate the number of channels and the maximum current level. ${ }^{65,66}$ The open probability was calculated as the ratio between the corrected macroscopic maximum current and the estimated maximum current calculated by the noise analysis of the whole-cell recordings.

\section{DATA AVAILABILITY}

Structure coordinates and cryo-EM density maps have been deposited in the protein data bank under accession numbers 7CR0 and EMD-30443 for apo KCNQ2, 7CR3 and EMD-30446 for apo KCNQ2-CaM, 7CR1 and EMD-30444 for ztz240-bound KCNQ2-Z, 7CR4 and EMD-30447 for ztz240-bound KCNQ2-CaM-Z, 7CR2 and EMD-30445 for RTG-bound KCNQ2-R, 7CR7 and EMD-30448 for RTG-bound KCNQ2-CaM-R.

\section{ACKNOWLEDGEMENTS}

Single-particle cryo-EM data were collected at Center of Cryo-Electron Microscopy at Zhejiang University. We are grateful to Dr. Xing Zhang, Shenghai Chang, and Xiaokang Zhang for their support in facility access and data acquisition. We thank Dr. Fan Yang and Lizhen $\mathrm{Xu}$ for their assistance with the noise analysis of the electrophysiological data. We thank the support of ECNU Multifunctional Platform for Innovation (001). This work was supported in part by the Ministry of Science and Technology of China (2018YFA0508100 to J.G. and Q.Z., and 2016YFA0500404 to S.Y.), the National Natural Science Foundation of China (31870724 to J.G., 31800699 to Q.Z., 31525001 and 31430019 to S.Y.), the Fundamental Research Funds for the Central Universities (to J.G. and H.Y.), the "Personalized Medicines-Molecular Signature-based Drug Discovery and Development", the Strategic Priority Research Program of the Chinese Academy of Sciences (XDA12040220 to H.Y.), the National Science and Technology Major Project "Key New Drug Creation and Manufacturing Program" of China (2018ZX09711002 to Q.Z.), and the "XingFuZhiHua" funding of ECNU (4430019311-542500/006 to H.Y.). 


\section{AUTHOR CONTRIBUTIONS}

J.G., H.Y. and Q.Z. conceived and designed this project. X.L., J.W., D.Lai, D.Lv, S.Y. and J.G. prepared the samples, and performed data acquisition, image processing and structure determination; Q.Z., P.G., J.F., L.M. and H.Y. did electrophysiological recording. All authors participated in the data analysis and manuscript preparation.

\section{ADDITIONAL INFORMATION}

Supplementary information accompanies this paper at https://doi.org/10.1038/ s41422-020-00410-8.

Competing interests: The authors declare no competing interests.

\section{REFERENCES}

1. Wang, H.-S. et al. KCNQ2 and KCNQ3 potassium channel subunits: molecular correlates of the M-channel. Science 282, 1890-1893 (1998).

2. Schroeder, B. C., Kubisch, C., Stein, V. \& Jentsch, T. J. Moderate loss of function of cyclic-AMP-modulated KCNQ2/KCNQ3 K+ channels causes epilepsy. Nature 396, 687-690 (1998)

3. Singh, N. A. et al. A novel potassium channel gene, KCNQ2, is mutated in an inherited epilepsy of newborns. Nat. Genet. 18, 25-29 (1998).

4. Charlier, C. et al. A pore mutation in a novel KQT-like potassium channel gene in an idiopathic epilepsy family. Nat. Genet. 18, 53-55 (1998).

5. Kato, M. et al. Clinical spectrum of early onset epileptic encephalopathies caused by KCNQ2 mutation. Epilepsia 54, 1282-1287 (2013).

6. Orhan, G. et al. Dominant-negative effects of KCNQ2 mutations are associated with epileptic encephalopathy. Ann. Neurol. 75, 382-394 (2014).

7. Weckhuysen, S. et al. KCNQ2 encephalopathy: emerging phenotype of a neonatal epileptic encephalopathy. Ann. Neurol. 71, 15-25 (2012).

8. Wulff, H., Castle, N. A. \& Pardo, L. A. Voltage-gated potassium channels as therapeutic targets. Nat. Rev. Drug Discov. 8, 982-1001 (2009).

9. Jentsch, T. J. Neuronal KCNQ potassium channels: physislogy and role in disease. Nat. Rev. Neurosci. 1, 21-30 (2000).

10. Soldovieri, M. V., Miceli, F. \& Taglialatela, M. Driving with no brakes: molecular pathophysiology of Kv7 potassium channels. Physiology 26, 365-376 (2011).

11. Stott, J. B., Jepps, T. A. \& Greenwood, I. A. KV7 potassium channels: a new therapeutic target in smooth muscle disorders. Drug Discov. Today 19, 413-424 (2014).

12. Zheng, Q. et al. Suppression of KCNQ/M (Kv7) potassium channels in dorsal root ganglion neurons contributes to the development of bone cancer pain in a rat model. Pain 154, 434-448 (2013).

13. Zhang, F. et al. Suppression of $K C N Q / M$ potassium channel in dorsal root ganglia neurons contributes to the development of osteoarthritic pain. Pharmacology 103, 257-262 (2019).

14. $\mathrm{Yu}, \mathrm{T}$. et al. KCNQ2/3/5 channels in dorsal root ganglion neurons can be therapeutic targets of neuropathic pain in diabetic rats. Mol. Pain. 14, 1-15 (2018).

15. Blackburn-Munro, G. \& Jensen, B. S. The anticonvulsant retigabine attenuates nociceptive behaviours in rat models of persistent and neuropathic pain. Eur. J. Pharmacol. 460, 109-116 (2003).

16. Munro, G. \& Dalby-Brown, W. Kv7 (KCNQ) channel modulators and neuropathic pain. J. Med. Chem. 50, 2576-2582 (2007).

17. Szelenyi, I. Flupirtine, a re-discovered drug, revisited. Inflamm. Res. 62, 251-258 (2013).

18. Wen, H. \& Levitan, I. B. Calmodulin is an auxiliary subunit of KCNQ2/3 potassium channels. J. Neurosci. 22, 7991-8001 (2002).

19. Bernardo-Seisdedos, G. et al. Structural basis and energy landscape for the Ca2+ gating and calmodulation of the Kv7. $2 \mathrm{~K}+$ channel. Proc. Natl. Acad. Sci. USA 115, 2395-2400 (2018).

20. Etxeberria, A. et al. Calmodulin regulates the trafficking of KCNQ2 potassium channels. FASEB J. 22, 1135-1143 (2008).

21. Zhang, Q. et al. Dynamic PIP2 interactions with voltage sensor elements contribute to KCNQ2 channel gating. Proc. Natl. Acad. Sci. USA 110, 20093-20098 (2013).

22. Wickenden, A. D., Yu, W., Zou, A., Jegla, T. \& Wagoner, P. K. Retigabine, a novel anti-convulsant, enhances activation of KCNQ2/Q3 potassium channels. Mol. Pharmacol. 58, 591-600 (2000).

23. Main, M. J. et al. Modulation of $K C N Q 2 / 3$ potassium channels by the novel anticonvulsant retigabine. Mol. Pharmacol. 58, 253-262 (2000).

24. Stafstrom, C. E., Grippon, S. \& Kirkpatrick, P. Ezogabine (retigabine). Nat. Rev. Drug Discov. 10, 729-730 (2011)

25. Friedel, H. A. \& Fitton, A. Flupirtine: a review of its pharmacological properties, and therapeutic efficacy in pain states. Drugs 45, 548-569 (1993).
26. Wickenden, A. D. et al. N-(6-chloro-pyridin-3-yl)-3, 4-difluoro-benzamide (ICA27243): a novel, selective KCNQ2/Q3 potassium channel activator. Mol. Pharmacol. 73, 977-986 (2008).

27. Padilla, K., Wickenden, A. D., Gerlach, A. C. \& McCormack, K. The KCNQ2/3 selective channel opener ICA-27243 binds to a novel voltage-sensor domain site. Neurosci. Lett. 465, 138-142 (2009).

28. Daniluk, J., Cooper, J. A., Stender, M. \& Kowalczyk, A. Survey of physicians' understanding of specific risks associated with retigabine. Drugs Real World Outcomes 3, 155-163 (2016).

29. Groseclose, M. R. \& Castellino, S. An investigation into retigabine (ezogabine) associated dyspigmentation in rat eyes by MALDI imaging mass spectrometry. Chem. Res. Toxicol. 32, 294-303 (2019).

30. Sun, J. \& MacKinnon, R. Cryo-EM structure of a KCNQ1/CaM complex reveals insights into congenital long QT syndrome. Cell 169, 1042-1050 (2017).

31. Sun, J. \& MacKinnon, R. Structural basis of human KCNQ1 modulation and gating. Cell 180, 340-347 (2020).

32. Gao, Z. et al. Isoform-specific prolongation of Kv7 (KCNQ) potassium channel opening mediated by new molecular determinants for drug-channel interactions. J. Biol. Chem. 285, 28322-28332 (2010).

33. Strulovich, R., Tobelaim, W. S., Attali, B. \& Hirsch, J. A. Structural insights into the M-channel proximal C-terminus/calmodulin complex. Biochemistry 55, 5353-5365 (2016).

34. Tao, X., Lee, A., Limapichat, W., Dougherty, D. A. \& Mackinnon, R. A gating charge transfer center in voltage sensors. Science 328, 67-73 (2010).

35. Li, P. et al. The gating charge pathway of an epilepsy-associated potassium channel accommodates chemical ligands. Cell Res. 23, 1106-1118 (2013).

36. Schenzer, A. et al. Molecular determinants of KCNQ (Kv7) K+ channel sensitivity to the anticonvulsant retigabine. J. Neurosci. 25, 5051-5060 (2005).

37. Wuttke, T. V., Seebohm, G., Bail, S., Maljevic, S. \& Lerche, H. The new anticonvulsant retigabine favors voltage-dependent opening of the Kv7.2 (KCNQ2) channel by binding to its activation gate. Mol. Pharmacol. 67, 1009-1017 (2005).

38. Lange, W. et al. Refinement of the binding site and mode of action of the anticonvulsant Retigabine on KCNQ K+ channels. Mol. Pharmacol. 75, 272-280 (2009).

39. Kim, R. Y. et al. Atomic basis for therapeutic activation of neuronal potassium channels. Nat. Commun. 6, 8116 (2015).

40. Gamper, N., Li, Y. \& Shapiro, M. S. Structural requirements for differential sensitivity of KCNQ K+ channels to modulation by Ca2+/calmodulin. Mol. Biol. Cell 16, 3538-3551 (2005).

41. Peretz, A. et al. Targeting the voltage sensor of Kv7.2 voltage-gated $\mathrm{K}+$ channels with a new gating-modifier. Proc. Natl. Acad. Sci. USA 107, 15637-15642 (2010).

42. Ottosson, N. E. et al. A drug pocket at the lipid bilayer-potassium channel interface. Sci. Adv. 3, e1701099 (2017).

43. Ahuja, S. et al. Structural basis of Nav1.7 inhibition by an isoform-selective smallmolecule antagonist. Science 350, aac5464 (2015).

44. $\mathrm{Xu}, \mathrm{H}$. et al. Structural basis of Nav1. 7 inhibition by a gating-modifier spider toxin. Cell 176, 702-715 (2019).

45. Shen, H., Liu, D., Wu, K., Lei, J. \& Yan, N. Structures of human Nav1.7 channel in complex with auxiliary subunits and animal toxins. Science 363, 1303-1308 (2019).

46. Clairfeuille, T. et al. Structural basis of a-scorpion toxin action on Nav channels. Science 363, eaav8573 (2019).

47. Hong, L., Kim, I. H. \& Tombola, F. Molecular determinants of Hv1 proton channel inhibition by guanidine derivatives. Proc. Natl. Acad. Sci. USA 111, 9971-9976 (2014).

48. Wang, A. W., Yang, R. \& Kurata, H. T. Sequence determinants of subtype-specific actions of KCNQ channel openers. J. Physiol. 595, 663-676 (2017).

49. Diver, M. M., Cheng, Y. \& Julius, D. Structural insights into TRPM8 inhibition and desensitization. Science 365, 1434-1440 (2019).

50. Song, K. et al. Structural basis for human TRPC5 channel inhibition by two distinct inhibitors. bioRxiv https://doi.org/10.1101/2020.04.21.052910 (2020).

51. Zhao, Y. et al. Molecular basis for ligand modulation of a mammalian voltagegated Ca2+ channel. Cell 177, 1495-1506 (2019).

52. Li, Y., Gamper, N., Hilgemann, D. W. \& Shapiro, M. S. Regulation of Kv7 (KCNQ) K+ channel open probability by phosphatidylinositol 4,5-bisphosphate. J. Neurosci. 25, 9825-9835 (2005).

53. Kumar, M. et al. Synthesis and evaluation of potent KCNQ2/3-specific channel activators. Mol. Pharmacol. 89, 667-677 (2016).

54. Shi, S. et al. Molecular mechanisms and structural basis of retigabine analogues in regulating KCNQ2 channel. J. Memb. Biol. 253, 167-181 (2020).

55. Wang, L., Qiao, G., Hu, H., Gao, Z. \& Nan, F. Discovery of novel retigabine derivatives as potent KCNQ4 and KCNQ5 channel agonists with improved specificity. ACS Med. Chem. Lett. 10, 27-33 (2019).

56. Morales-Perez, C. L., Noviello, C. M. \& Hibbs, R. E. Manipulation of subunit stoichiometry in heteromeric membrane proteins. Structure 24, 797-805 (2016). 
57. Zheng, S. Q. et al. MotionCor2: anisotropic correction of beam-induced motion for improved cryo-electron microscopy. Nat. Methods 14, 331-332 (2017).

58. Zhang, K. Gctf: real-time CTF determination and correction. J. Struct. Biol. 193 1-12 (2016).

59. Scheres, S. H. RELION: implementation of a Bayesian approach to cryo-EM structure determination. J. Struct. Biol. 180, 519-530 (2012).

60. Emsley, P., Lohkamp, B., Scott, W. G. \& Cowtan, K. Features and development of Coot. Acta Crystallogr. D Biol. Crystallogr. 66, 486-501 (2010).

61. Adams, P. D. et al. PHENIX: a comprehensive Python-based system for macromolecular structure solution. Acta Crystallogr. D Biol. Crystallogr. 66, 213-221 (2010).
62. Chen, V. B. et al. MolProbity: all-atom structure validation for macromolecular crystallography. Acta Crystallogr. D Biol. Crystallogr. 66, 12-21 (2010).

63. Schrodinger, L. The PyMOL molecular graphics system. Version 1.8 (2015).

64. Pettersen, E. F. et al. UCSF Chimera-a visualization system for exploratory research and analysis. J. Comput. Chem. 25, 1605-1612 (2004).

65. Sigworth, F. J. The variance of sodium current fluctuations at the node of Ranvier. J. Physiol. 307, 97-129 (1980).

66. Yang, F., Cui, Y., Wang, K. \& Zheng, J. Thermosensitive TRP channel pore turret is part of the temperature activation pathway. Proc. Natl. Acad. Sci. USA 107, 7083-7088 (2010). 